\title{
Piezoelectric Material Based Technique for Concurrent Force Sensing and Energy Harvesting for Interactive Displays
}

\author{
Shuo Gao ${ }^{1}$, Chun-yen Huang ${ }^{2}$ and Linxiao $\mathrm{Wu}^{3}$ \\ ${ }^{1}$ Department of Electronic and Electrical Engineering, University College London, London, WC1E 7JE, UK \\ ${ }^{2}$ Department of Engineering, University of Cambridge, Cambridge, CB3 0FA, UK \\ ${ }^{3}$ Department of Nanotechnology, University College London, London, NW3 2QG, UK \\ \{email: linxiao.wu.13@ucl.ac.uk\}
}

\begin{abstract}
-3-dimensional touch sensing and long battery lifetime are two desired attributes in interactive displays. By employing piezoelectric material and rectifier circuitry related technique, force touch induced charges are used for both force strength detection and energy harvesting, achieving 3dimensional touch sensing and extended battery lifetime at the same time for interactive displays.
\end{abstract}

Keywords - Interactive display, piezoelectric material, force sensing and energy harvesting

\section{INTRODUCTION}

Long battery lifetime and 3-dimensional touch sensing are two practical and critical demands for today's electronic devices, especially for portable devices such as smart phones. In terms of the former demand, in smart phones, high power consumption, mainly caused by radio, display, and Wi-Fi components, shortens the battery lifetime, giving rise to the popularity of portable charging devices. Two means traditionally considered for extending battery lifetime are improvement of the capabilities of the battery, and reducing power consumption. 2-D materials such as graphene are used to increase the overlap area of the capacitor's [1]; and a variety of low power consumption techniques have been proposed and implemented [2]-[4] to efficiently reduce power consumption. However, these two approaches are constrained by the technology development, hence cannot fully satisfy user' need. Harvesting environmental energy is thus essential, and has attracted increasing attention. Traditional energy-harvesting techniques in smart phones are based on collecting RF, solar and thermoelectric related energy. However, RF energy may cause wave pollution [5], a strong sunlight environment is needed for collecting solar energy, and thermoelectric energy requires a stable heat source, which is not convenient for customers. As to the latter demand, although force touch sensing has been achieved [6], additional integrated components give rise to complexity circuit and extra power consumption. Thus, a green, convenient and simple structured technique is required for smart phones for both energy harvesting and force sensing.

In this work, a piezoelectric material based touch panel for simultaneously detecting force touch strength and harvesting

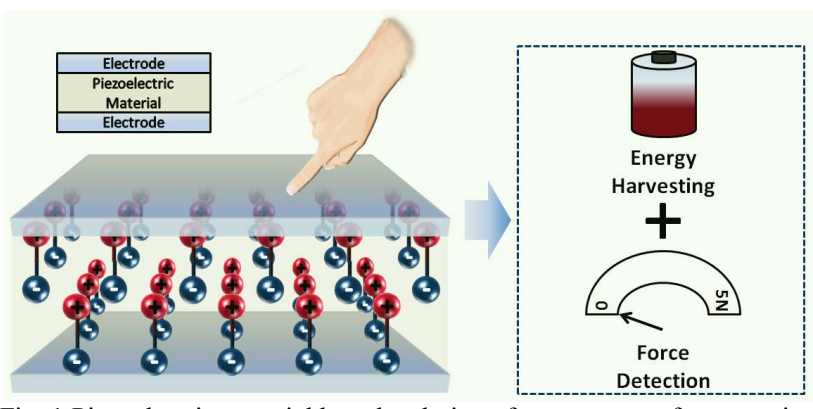

Fig. 1 Piezoelectric material based technique for concurrent force sensing and energy harvesting.

force induced electrical energy is presented for the first time. Experimental results from a sandwich structured stack-up (as conceptually shown in Fig 1, where piezoelectric material is used for force sensing and energy harvesting), shows that 3dimensional touch sensing ( $\mathrm{x}-\mathrm{y}-\mathrm{z})$ and enhanced battery lifetime are achieved at the same time, indicating a strong potential in providing customers with enhanced user experience.

\section{HARVESTED FORCE TOUCH INDUCED ELECTRICAL ENERGY}

In our previous work, piezoelectric material has been proposed for force touch detection in interactive displays, along with detailed mechanical analysis and experimental validation [7][8]. The force strength has a positive linear relationship with the induced voltage value. In scalar expression, the relationship is expressed as [7]

$$
V=t d_{33} F / A \varepsilon_{0} \varepsilon_{r}
$$

where $t$ and $A$ represent the piezoelectric film thickness and contact area, respectively. $d_{33}$ denote piezoelectric coefficient, $\varepsilon_{0}$ is vacuum permittivity and $\varepsilon_{\mathrm{r}}$ is the relative permittivity of piezoelectric material. Below, we first discuss how the force induced electrical energy can be stored in a capacitor which represents a battery, and then the technique for concurrent force detection and energy harvesting is proposed and implemented. 


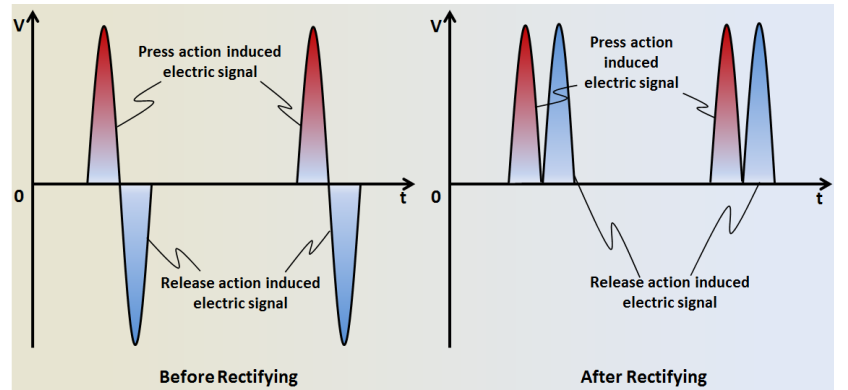

Fig. 2 Force touch induced electrical signal, before (left) and after (right) bridge rectifier circuit.

As conceptually shown in Fig. 2, the piezoelectric material based force induced electric signal has positive and negative components since the polarization changes twice during a press-and-release finger touch event. This kind of signal cannot be directly stored in an energy storage component such as a capacitor, because the negative part compensates for the positive part. A single Diode based rectifier circuit can be used to block the negative part. However, blocking the negative part wastes half of the force transferred energy. Thus, bridge rectifier circuits, are widely used to fully collect the energy. The collected energy in the capacitor, by tapping a polyvinylidene difluoride (PVDF) laminated capacitive touch panel (described in [7]), is shown in Fig. 3a, in which the charging and discharging periods are clearly shown. After each force touch, the capacitor charges a little bit. Zooming in on the charging period, there are two charging moments during a single force touch event. Because the polarization changes twice, the energy in the negative part is also collected after the bridge rectifier, and is used to charge the capacitor, as shown in Fig. $3 b$.

The following equation can be used to calculate the total energy stored in the capacitor:

$$
E_{E . H .}=\frac{1}{2} C V^{2}
$$

where $E_{E . H}$ is the energy stored in the capacitor, $\mathrm{C}$ denotes the capacitance of the capacitor, and $V$ represents the voltage across the capacitor. To calculate the stored energy in Fig. 3a, the following values are used for the variables in Eq. 2: $C$ is equal to $1 \mu \mathrm{F}$, and $\mathrm{V}$ is around $45 \mathrm{mV}$. Thus, based on the Eq. $2, E_{E . H}$. is equal to $1.01 \mathrm{~nJ}$. The following equation can be used to calculate the energy harvested by a single force touch:

$$
\Delta E_{E . H .}=\frac{1}{2} C \Delta V^{2}
$$

where $\Delta E_{E . H .}$ indicates the energy stored by a single force touch, and $\Delta V$ represents the voltage change after a single force touch.

It can be observed that the stored energy decays severer at higher voltage values, this phenomenon can be explained by:

$$
V(t)=V_{0}\left(e^{-t / \tau}\right)
$$

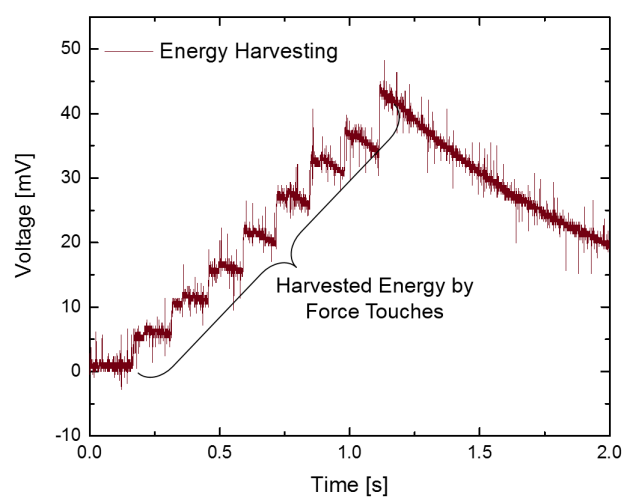

(a)

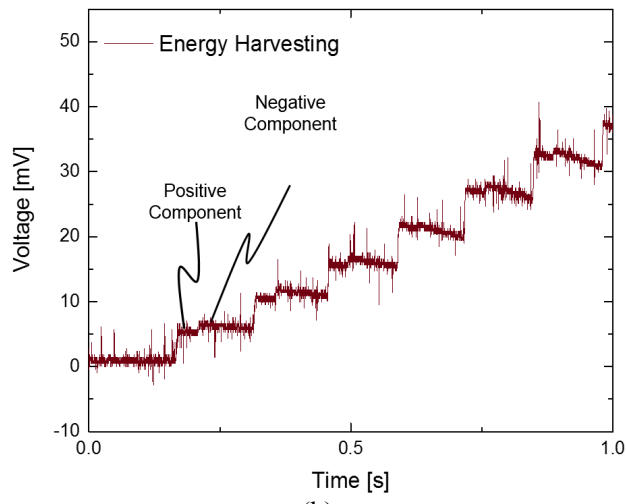

(b)

Fig. 3 Harvested force induced electrical energy.(a) Charging period by force touches (b) Zoom-in of a, demonstrating both positive and negative components are collected

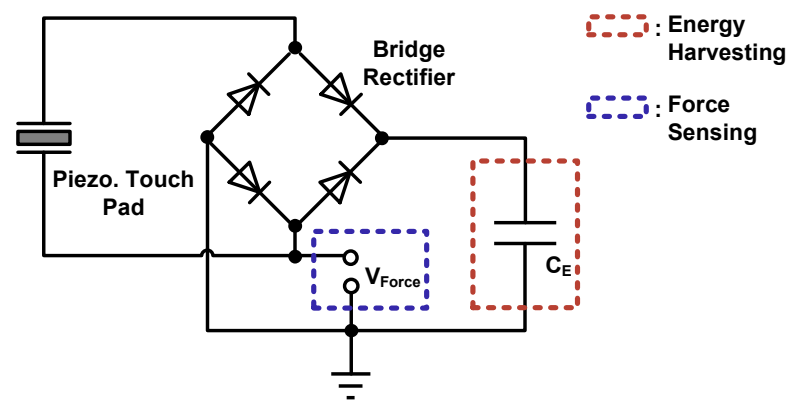

Fig. 4 Bridge rectifier based circuit for concurrently detecting force strength and harvesting force induced electrical energy.

here $V_{0}$ indicates the initial voltage value, $\tau$ is the time constant of the RC network and $V(t)$ denote the voltage value after time $t$.

\section{CONCURRENT ForCE SENSING AND ENERGY HARVESTING}

Above, we demonstrate that the force induced charges can be used to charge a capacitor, thus when the software application doesn't need to read force information, the force induced charge can be used for energy harvesting. However, it is more desired to obtain these two functions (force sensing and energy harvesting) at the same time. To achieve this, in the proposed technique, the positive part of the force induced 

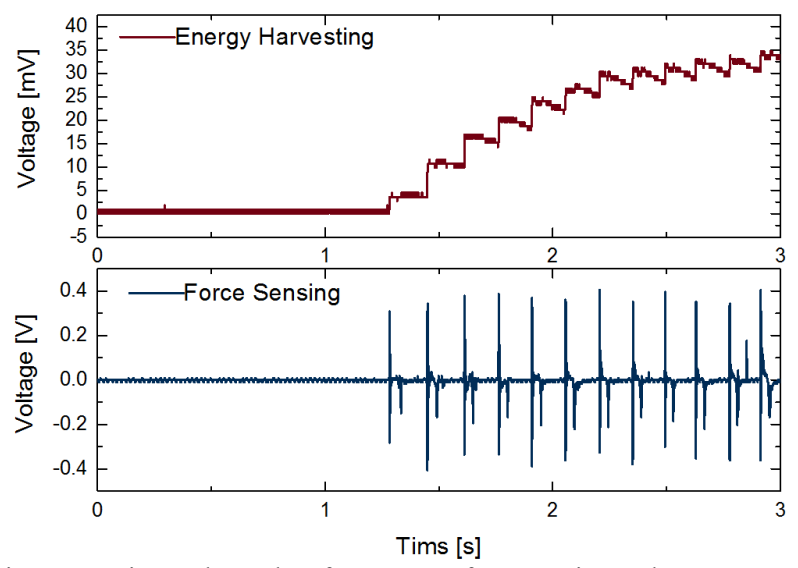

Fig. 5 Experimental Results of concurrent force sensing and energy harvesting.

electrical signal is used for force touch detection, and the negative part is employed for energy harvesting. A bridge rectifier based circuit (as shown in Fig. 4) is implemented here to realize concurrent force touch detection and energy harvesting.

Fig. 4 shows that the force detection circuit and energy harvesting circuit share the same ground. Both of them use half of the energy generated by force touches. To illustrate these two functions, the force detection circuit and the energy harvesting circuit are connected to an oscilloscope. The corresponding touch signal and energy harvesting signal are shown in Fig. 5, respectively. We can observe that force detection and energy harvesting are successfully achieved at the same time. A high force touch sensitivity of $0.24 \mathrm{~V} / \mathrm{N}$ is obtained and averagely each force touch can charge the capacitor $0.06 \mathrm{~nJ}$. In practical circuit design, a charge amplifier is widely used to read the force touch signal, due to the high impedance of the PVDF sample. Since the oscilloscope already equipped with high impedance, there was no charge amplifier used in this demonstration.

\section{CONCULUSION}

In this work, a technique for concurrent force touch sensing and energy harvesting is successfully proposed and implemented, by utilizing polarization change of piezoelectric material and rectifier related circuit design. With the presented technique, high force detection sensitivity $(0.24 \mathrm{~V} / \mathrm{N})$ and energy harvesting efficiency $(0.06 \mathrm{~nJ}$ per touch) are obtained at the same time. The work in this paper has significant meaning for interactive displays when high force touch sensitivity and long battery lifetime are required.

\section{REFERENCES}

[1] Y. Li, K. Yan, H-W Lee, Z. Lu, N. Liu and Y. Cui, "Growth of conformal graphene cages on micrometre-sized silicon particles as stable battery anodes", Nat. Energy, vol. 1, pp. 15029, 2016.

[2] S-M. Kim, H. Cho, M. Nam, S-G Choi and K. Cho, "Low-power touchsensing circuit with reduced scanning algorithm for touch screen panels on mobile devices", IEEE/OSA J. Display Technol., vol. 11, no. 1, pp. 36-43, 2015.

[3] I. Hong, K. Bong, D. Shin, S. Park, K. Lee, Y. Kim and H-J Yoo, "A $2.71 \mathrm{~nJ} /$ pixel 3D-stacked gaze-activated object-recognition system for low-power mobile HMD applications", IEEE Int. Solid-State Circuits Conf. Dig. Tech. Papers, San Francisco, USA, February 2015, pp. 326327.

[4] B. Liu, Z. Hoseini, K-S. Lee and Y-M Lee, "On-Chip touch sensor readout circuit using passive sigma-delta modulator capacitance-todigital converter", IEEE Sens. J, vol. 15, no. 7, pp. 3893-3902, 2015.

[5] A. Balmori, "Electromagnetic pollution from phone masts. Effects on wildlife", Pathophysiology, vol. 16, no. 2, pp. 191-199, 2009.

[6] Y-T. Kim, J-H Kim, D-K Kim and Y-H Kwon, "Force sensing model of capacitive hybrid touch sensor using thin-film force sensor and its evaluation", Int. J. Precis. Eng. Man., vol. 16, no. 5, pp. 981-988, 2015.

[7] S. Gao, V. Arcos and A. Nathan, "Piezoelectric vs. capacitive based force sensing in capacitive touch panels", IEEE Access, vol. 4, pp. 37693774, 2016.

[8] S. Gao, X. Wu, H. Ma, J. Robertson and A. Nathan, "Ultrathin Multifunctional Graphene-PVDF Layers for Multidimensional Touch Interactivity for Flexible Displays", ACS Appl. Mater. Interfaces, vol. 9, no. 22, pp. 18410-18416, 2017. 